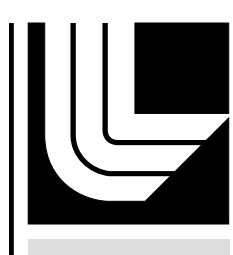

LAWRENCE LIVERMORE N A T IO N A L LABORATORY

\title{
Heavy Ion Inertial Fusion Energy: Summaries of Program Elements
}

A. Friedman, J. J. Barnard, I. Kaganovich, P. A. Seidl, R. J. Briggs, A. Faltens, J. W. Kwan, E. P. Lee, B. G. Logan

March 3, 2011 
This document was prepared as an account of work sponsored by an agency of the United States government. Neither the United States government nor Lawrence Livermore National Security, LLC, nor any of their employees makes any warranty, expressed or implied, or assumes any legal liability or responsibility for the accuracy, completeness, or usefulness of any information, apparatus, product, or process disclosed, or represents that its use would not infringe privately owned rights. Reference herein to any specific commercial product, process, or service by trade name, trademark, manufacturer, or otherwise does not necessarily constitute or imply its endorsement, recommendation, or favoring by the United States government or Lawrence Livermore National Security, LLC. The views and opinions of authors expressed herein do not necessarily state or reflect those of the United States government or Lawrence Livermore National Security, LLC, and shall not be used for advertising or product endorsement purposes.

This work performed under the auspices of the U.S. Department of Energy by Lawrence Livermore National Laboratory under Contract DE-AC52-07NA27344. 


\title{
Heavy Ion Inertial Fusion Energy: Summaries of Program Elements*
}

\begin{abstract}
Alex Friedman, Lawrence Livermore National Laboratory John J. Barnard, Lawrence Livermore National Laboratory Igor Kaganovich, Princeton Plasma Physics Laboratory Peter A. Seidl, Lawrence Berkeley National Laboratory Richard J. Briggs, Science Applications International Corp. Andris Faltens, Lawrence Berkeley National Laboratory Joe W. Kwan, Lawrence Berkeley National Laboratory Edward P. Lee, Lawrence Berkeley National Laboratory B. Grant Logan, Lawrence Berkeley National Laboratory
\end{abstract}

A collection of brief summaries, prepared in response to the Request for Information on Topical Areas in Inertial Fusion Energy that was issued by the National Academy Of Sciences' Committee on the Prospects for Inertial Confinement Fusion Energy Systems, February 3, 2011.

Included herein:

\section{HEAVY ION INERTIAL FUSION ENERGY HEAVY ION ACCELERATOR DRIVERS FINAL OPTICS -- HEAVY ION BEAMS BEAM PLASMA INTERACTIONS}

February 28, 2011

\footnotetext{
* This work was performed under the auspices of the U.S. Department of Energy by Lawrence Livermore National Security, LLC, Lawrence Livermore National Laboratory under Contract DE-AC52-07NA27344, by LBNL under Contract DE-AC02-05CH11231, and by PPPL under Contract DE-AC02-76CH03073.
} 



\section{HEAVY ION INERTIAL FUSION ENERGY}

\section{Description}

The goal of the Heavy Ion Fusion (HIF) Program is to apply high-current accelerator technology to IFE power production. Ion beams of mass $\sim 100 \mathrm{amu}$ and kinetic energy $\geq 1 \mathrm{GeV}$ provide efficient energy coupling into matter, and HIF enjoys R\&D-supported favorable attributes of: - the driver, projected to be robust and efficient; see "Heavy Ion Accelerator Drivers."

- the targets, which span a continuum from full direct to full indirect drive (and perhaps fast ignition), \& have metal exteriors that enable injection at $\sim 10 \mathrm{~Hz}$; see "IFE Target Designs."

- the near-classical ion energy deposition in the targets; see "Beam-Plasma Interactions."

- the magnetic final lens, robust against damage; see "Final Optics-Heavy Ion Beams."

- the fusion chamber, which may use neutronically-thick liquids; see "Liquid-Wall Chambers." Most studies of HIF power plants have assumed indirect drive and thick liquid wall protection, but other options are possible.

Status

- Existing accelerators are comparable to projected HIF drivers in size, cost, total beam energy, focusing, average beam power, repetition rate, reliability, and durability. A peak power of 1001000 TW shared over $\sim 100$ beams is the new requirement; use of $\sim 10 \mathrm{x}$ final pulse compression reduces the required current at accelerator exit to $\sim 0.1-1 \mathrm{TW} /$ beam. For reference, CERN's ISR had a single-beam power (protons) of $1 \mathrm{TW}$ at $30 \mathrm{GeV}$. Fig. 1 shows a typical driver layout.

- A variety of heavy-ion-driven target designs have been developed and simulated in 2-D.These include 2-sided radiation-driven targets resembling those to be tested on NIF, but without laser entrance holes and with converters that absorb ions and produce x-rays. See Fig. 2.

- Successful experiments, along with simulations, have addressed the most important driverbeam manipulations (Figs. 3, 4); the results support projections of beam intensity on target. With currents ranging from $1 \mathrm{~mA}$ to $1 \mathrm{~A}$, these scaled experiments had correct, driver-like dimensionless parameters, e.g., "tune depression" (defocusing due to beam space charge) and perveance (ratio of space charge potential energy to ion beam kinetic energy). The Neutralized Drift Compression Experiment-II (NDCX-II) being built at LBNL (Fig. 5) will reach 100A on target, important to its mission of rapidly heating foil targets before they expand.

Studies of heavy ion power plants predict a cost of electricity similar to that of other fusion options, with additional benefits of long life and minimal use of exotic first-wall materials.

\section{R\&D Goals and Challenges}

\section{Current Research and Development (R\&D)}

- Extend validation of beam acceleration and focusing at high current, and for multiple beams. Continue source-through-target simulation studies for each step toward a power plant system.

- Optimize target design to minimize required driver cost and beam focusing requirements.

- Work with industry to further develop and reduce the cost of custom accelerator components.

- Contribute to and learn from fusion, plasma, and accelerator science and technology.

\section{Related R\&D Activities}

- The DOE/SC/FES program on ion-heated HEDLP / Warm Dense Matter physics.

- The research programs in heavy ion fusion and related areas, in Europe, Russia, and Japan.

- The large worldwide research programs in accelerators for a broad range of applications.

- The ICF program in targets; the MFE program (esp. work on plasma simulation and magnets).

- The high-perveance beam physics experiments at U. MD (UMER) and Princeton (PTSX).

- The pulsed power fusion program, particularly for chamber protection and power handling. Recent Successes

- Experimental demonstration of: focusing to mm-scale spots; beam merging for a compact 
injector and as a driver manipulation; and target injection with accuracy for indirect drive.

- Design and near-completion of NDCX-II, a new short-pulse ion beam facility, motivated initially for HEDLP / Warm Dense Matter, but well suited for HIF studies. NDCX-II is a prerequisite for a future facility on the DOE/SC list, the Integrated Beam-HEDP experiment.

- Solid progress toward validated simulations, including a 3D PIC code for HIF beam dynamics.

Anticipated Contributions

\section{Metrics}

- A design for an efficient, reliable, durable, low-cost, and environmentally attractive driver and focusing system having adequate repetition rate.

- A target design optimized for yield, cost, and driver requirements, manufacturable in quantity.

- A well-defined, affordable development path involving acceptable levels of risk at each step.

Near Term (first $\sim 5$ years)

- Complete and operate NDCX-II to validate predictions of hardware and ion beam performance.

- Extend NDCX-II; add a non-neutral drift line, bend, and quadrupole final focus, and use to study ion beam compression, bending, and focusing with driver-like dimensionless parameters

- Upgrade HCX for $\sim 5 \mathrm{~Hz}$ operation, and extend to answer long path length questions; validate predicted dynamics of its driver-scale beam to enable design of next-step machine.

- Improve key technologies: quadrupole arrays, pulsers, insulators, ferromagnetic-core materials.

- Develop designs for several target options; monitor developments in laser-driven targets.

- Partner in a systematic program of scaled experiments on liquid-wall chambers.

- Extend studies of integrated driver/target/balance-of-plant.

Midterm (next $\sim 10$ years)

- Develop, construct and operate $\sim 10-100 \mathrm{~kJ}$ Heavy-Ion-Driven Implosion Experiment (HIDIX).

- Use HIDIX for definitive experiments in beam acceleration and control, focusing, chamber physics, and those aspects of ion target physics that cannot be done on existing laser facilities.

- Continue development of key technologies listed above, for next phase.

- Conduct liquid chamber, target fabrication, and injection R\&D for $5 \mathrm{~Hz}$ experiments on HIDIX.

- Develop engineering design for a full-scale driver.

Long Term (next $\sim 20$ years)

- Build 2-3 MJ HIF ignition test facility for single shots, then burst mode, using 5-Hz driver.

- Complete full-scale chamber experiments.

- Add nuclear systems; upgrade to 150 MW average-fusion-power HIF DEMO (same driver).

\section{Principal steps to a DEMO plant}

(NDCX-II \& upgraded HCX) $\rightarrow$ HIDIX $\rightarrow$ HIF ignition facility $\rightarrow$ upgrade to DEMO.

\section{Proponents' Claims}

- The demonstrated technology and known costs of accelerators carry over directly to HIF IFE.

- Past experiments and simulations give confidence in attaining focused-beam intensities.

- Heavy-ion drivers are efficient enough to allow either indirect or direct drive target IFE targets.

- Heavy-ion energy coupling into targets is effective and does not entail collective scattering.

- Focusing magnets can be long-lived when adequately shielded from line-of-sight target output.

- Ion beam transport in the chamber is compatible with the vapor pressure of the internal liquid.

- Thick liquid protection shields the first wall and reduces the required magnet coil shielding.

- The ignition test facility driver can be re-used for a DEMO plant, reducing cost, risk, and time.

\section{Critics' Claims}

- Risk or delay in the program may be engendered by the modest scale of most HIF experiments.

- A heavy ion driver will be expensive, and/or the development steps costly.

- Beam focusing / quality requirements will impose additional costs or require too many beams. 


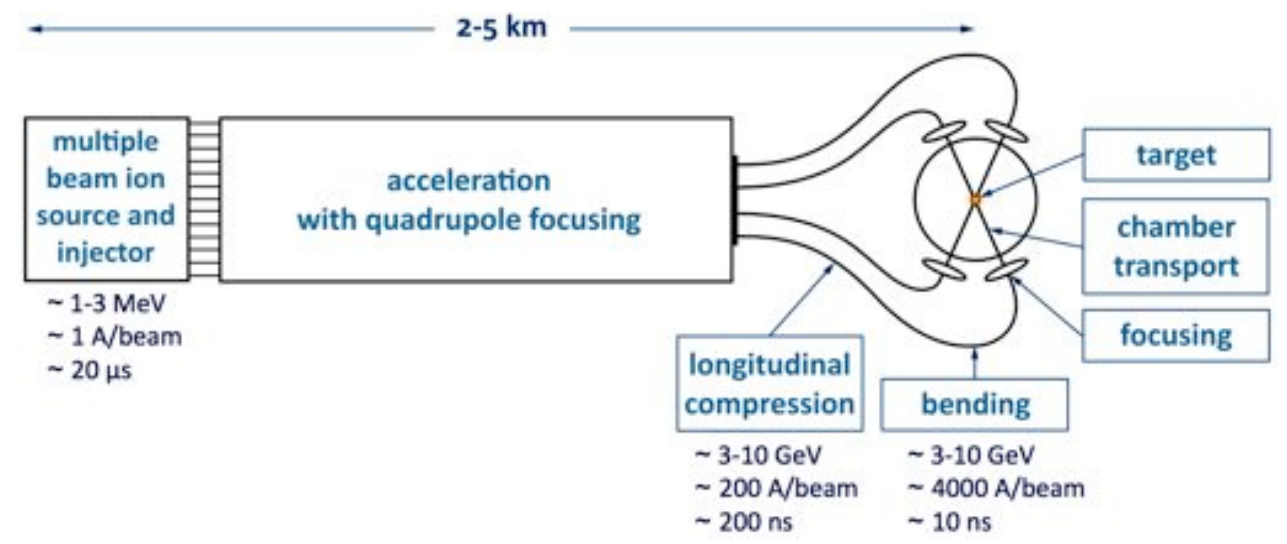

Fig. 1. Schematic representation of a typical HIF driver, final optics, and fusion chamber.

(a)

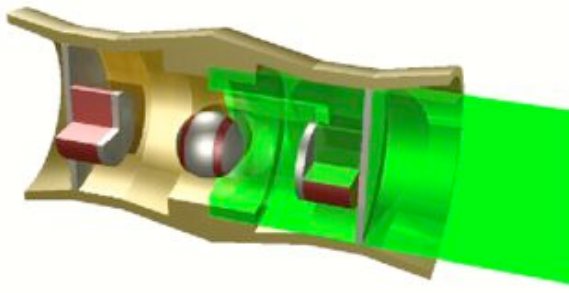

(b)

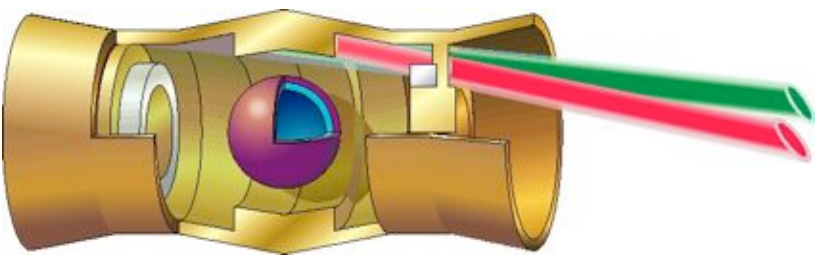

Fig. 2. Indirect-drive HIF targets: (a) "Hybrid" with $\mathrm{Pb}^{+}$beam at 3-4.5 GeV, beam spot $3.8 \times 5.4 \mathrm{~mm}$, 6.7 MJ input, gain 58; and (b) "Distributed Radiator" with $\mathrm{Pb}^{+}$beam at 3.3-4 GeV, beam spot $1.8 \mathrm{x}$ $4.1 \mathrm{~mm}, 5.9$ MJ input, gain 68 [D. Callahan et al., Laser and Particle Beams (2002)].

(a)

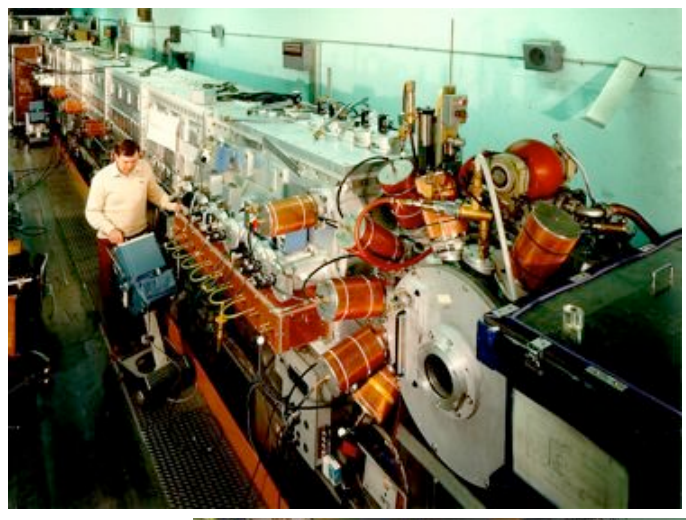

(b)

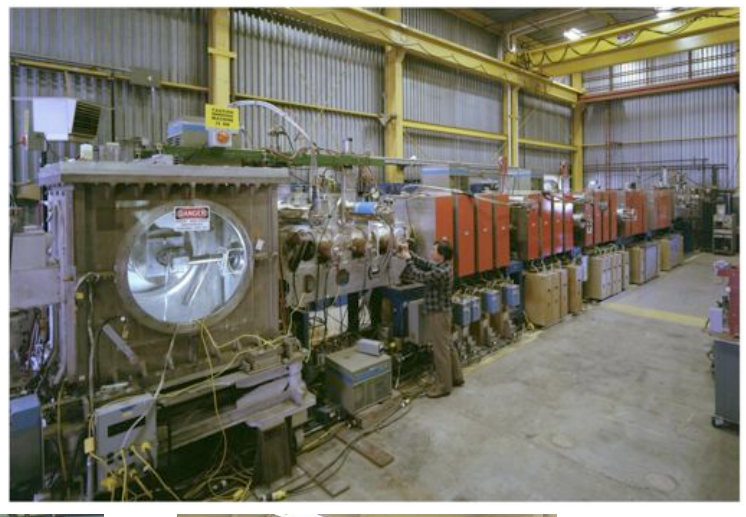

(c)

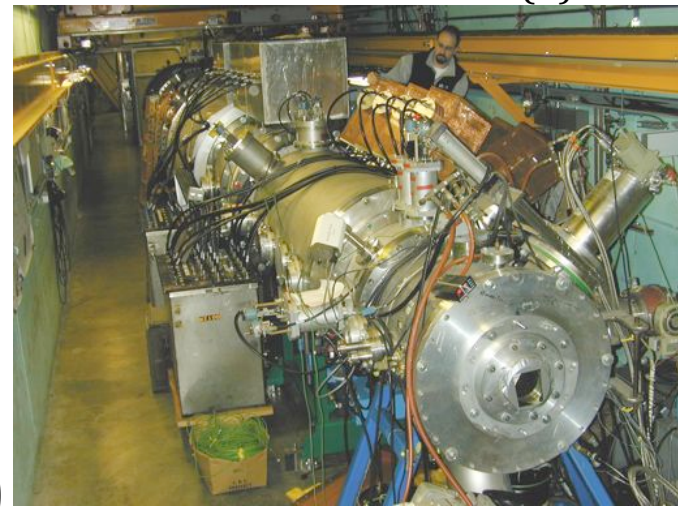

(d)

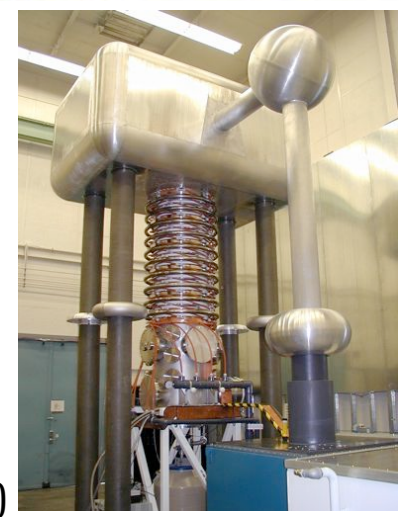

Fig. 3. (a) Single-Beam Transport Experiment (SBTE), (b) Multiple-Beam Experiment-4 (MBE-4), (c) High-Current Experiment (HCX), all at LBNL; (d) 500-kV injector test stand at LLNL. 

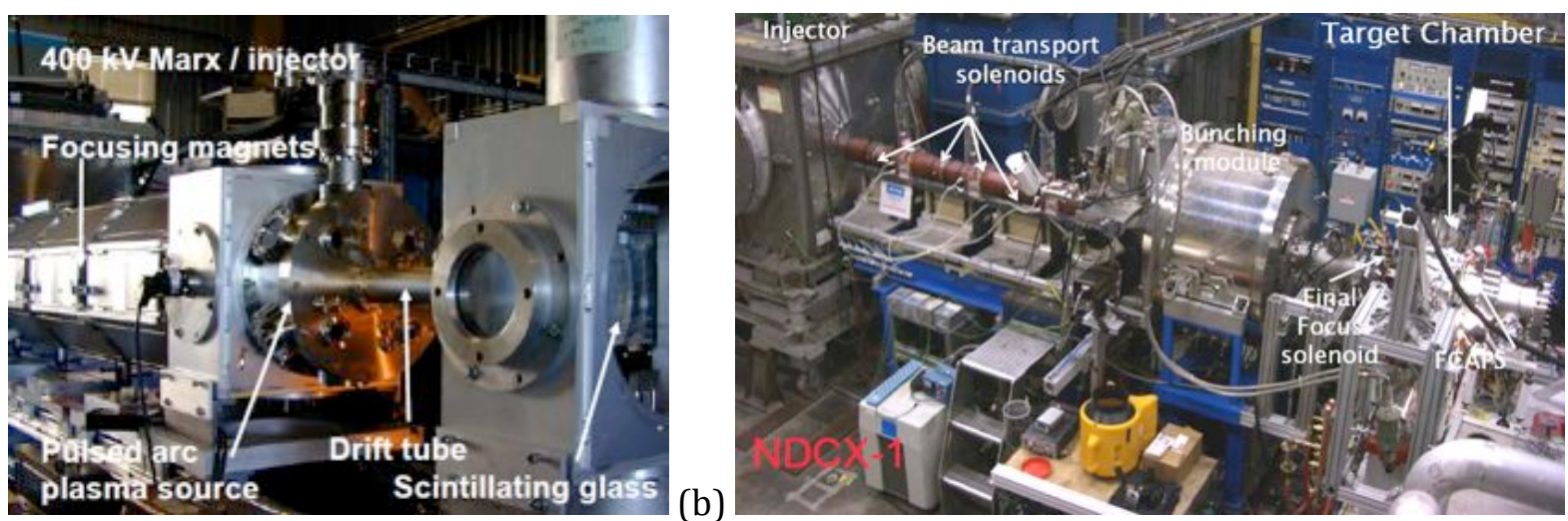

Fig. 4. (a) Neutralized Transport Experiment (NTX), and (b) Neutralized Drift Compression Experiment-I (NDCX-I), both at LBNL.

(a)
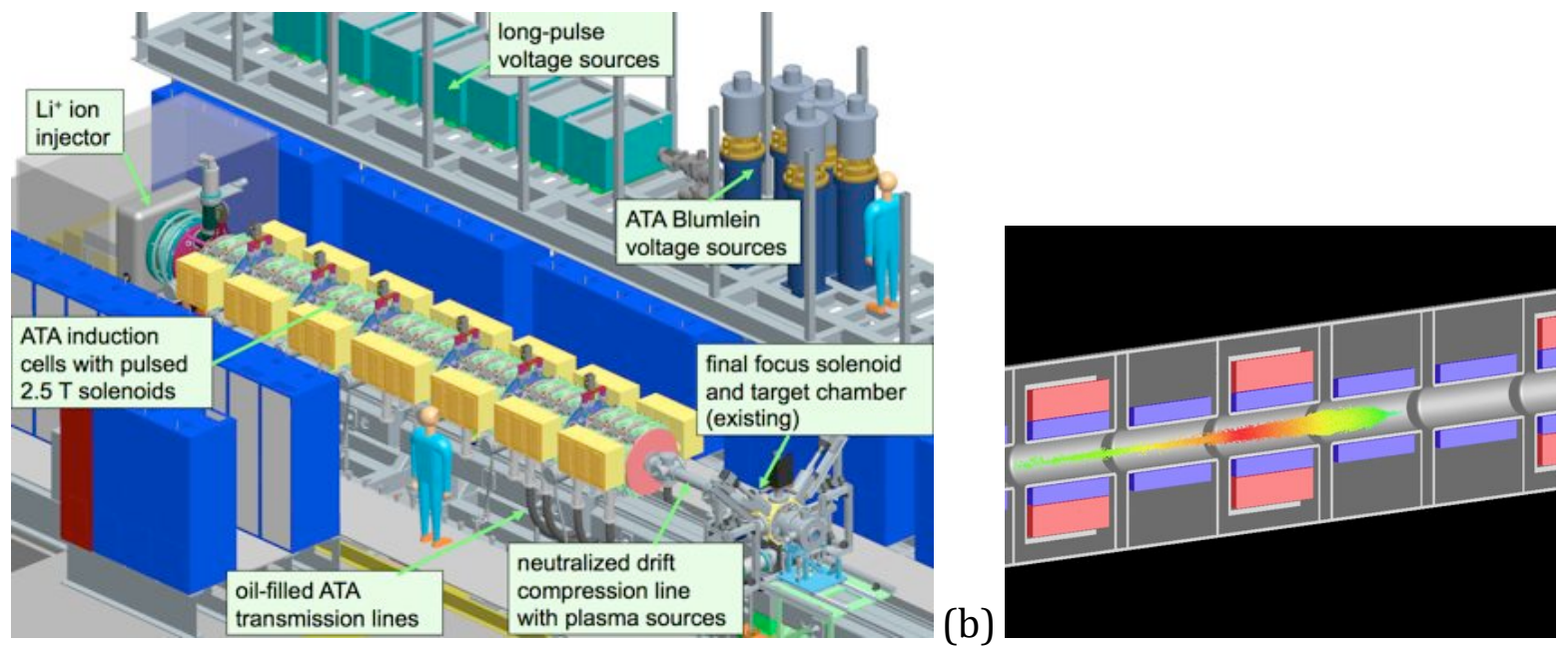

(c)
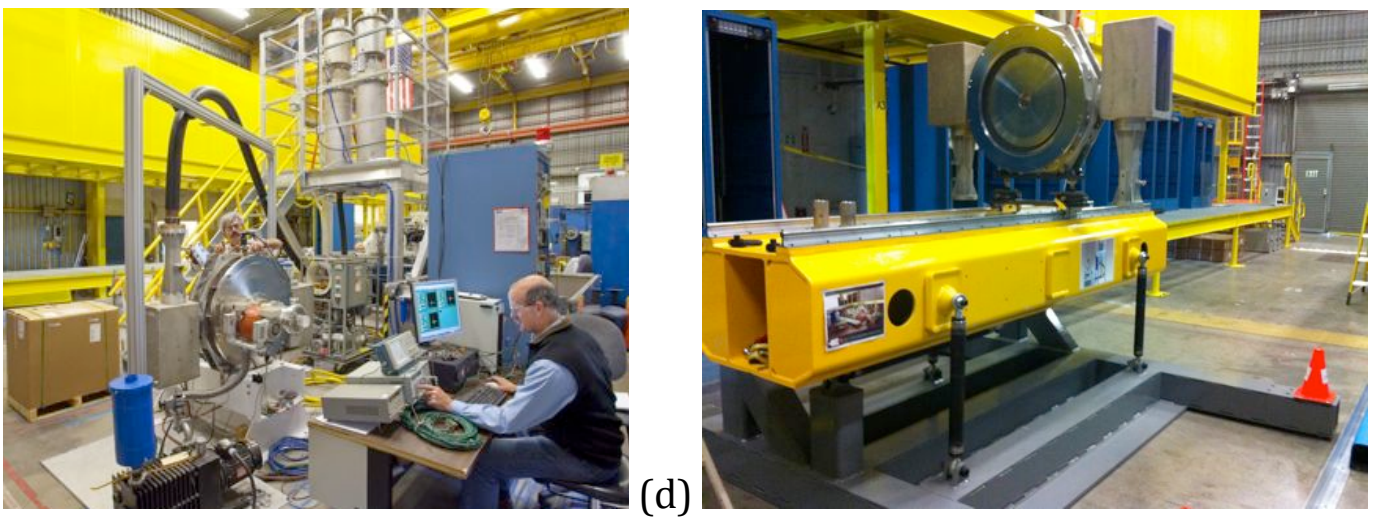

Fig. 5. NDCX-II: (a) initial configuration, (b) 3D PIC simulation of beam dynamics, (c) test stand, and (d) first support-rail segment, an induction cell, and equipment racks as of February 9, 2011. 


\section{Authors and Contributors}

\section{Principal Author:}

Alex Friedman, Lawrence Livermore National Laboratory

\section{Co-Authors:}

John J. Barnard, Lawrence Livermore National Laboratory Igor Kaganovich, Princeton Plasma Physics Laboratory Joe W. Kwan, Lawrence Berkeley National Laboratory B. Grant Logan, Lawrence Berkeley National Laboratory Peter A. Seidl, Lawrence Berkeley National Laboratory

\section{Contributors:}

Andris Faltens, Lawrence Berkeley National Laboratory Edward P. Lee, Lawrence Berkeley National Laboratory Richard M. More, Lawrence Berkeley National Laboratory William L. Waldron, Lawrence Berkeley National Laboratory

Ronald H. Cohen, Lawrence Livermore National Laboratory William M. Sharp, Lawrence Livermore National Laboratory Matthew R. Terry, Lawrence Livermore National Laboratory

Erik Gilson, Princeton Plasma Physics Laboratory

Larry Grisham, Princeton Plasma Physics Laboratory

Ed Startsev, Princeton Plasma Physics Laboratory

Dale R. Welch, Voss Scientific

David V. Rose, Voss Scientific

Irving Haber, University of Maryland

Rami A. Kishek, University of Maryland

David Sutter, University of Maryland

Richard J. Briggs, SAIC 



\section{Description}

Heavy ions of mass $\sim 100$ amu and ion kinetic energy $\geq 1 \mathrm{GeV}$ have a stopping range suitable to drive IFE targets with yield $>100 \mathrm{MJ}$ and gain $>50$. A heavy-ion driver must deliver $1-10 \mathrm{MJ}$ of energy, properly shaped, at a peak power $\geq 100 \mathrm{TW}$ at $\sim 10 \mathrm{~Hz}$. The beams' quality and alignment must be such that they can be focused on the target to a radius of a few millimeters from a distance of several meters. Limitations due to space charge, emittance growth, beam-gas, and beam-plasma interactions must be sufficiently controlled throughout the driver. Because of the high charge per bunch, the general approach is to accelerate a longer bunch and then compress it to the short length required at the target. Nuclear and high energy physics accelerators, with total beam energy of $\geq 1 \mathrm{MJ}$ have separately exhibited intrinsic efficiencies, pulse repetition rates $(>100 \mathrm{~Hz})$, power levels $(\mathrm{TW})$, and durability required for IFE (Table 1).

\section{Status}

The three main types of heavy ion drivers are synchrotrons, RF linacs (with storage rings) and induction linacs. RF accelerators are appealing because of the extensive experience in high energy and nuclear physics; and induction accelerators, because of their much higher efficiency and higher particle beam current (10 kA in some applications). The US effort has focused on induction accelerators because of higher efficiency and because there is no need to accumulate charge in storage rings; their nonresonant character allows pulse compression. Since the beam space charge repulsion is large compared to the effective temperature, the mean transverse focusing force must balance the space charge force. To subdivide the $\sim 100 \mathrm{kA}$ impinging on the target to beams with a few kA each, a single accelerator for multiple beams was an early innovation to meet the focal spot requirements. The multiple beams can be accelerated simultaneously through the same induction cells, interspersed with arrays of superconducting magnets (Fig. 1). Tests of induction modules have shown efficiencies of up to $40 \%$. Integrated target designs and accelerator designs have been merged in consistent systems models.

\section{R\&D Goals and Challenges}

\section{Current Research and Development (R\&D)}

The challenge is to inject and maintain high beam brightness and low emittance through the accelerator and to the target despite the large space charge forces that dominate the beam dynamics for most of the accelerator. The beam emittance is the volume occupied by the particles of the beam in space and momentum phase space as it travels. Sources of emittance growth include imperfections of the accelerator focusing magnet fields, alignment, beam-gas interactions and the effect on the beam of unwanted electrons, chromatic aberrations, and beam-plasma interactions in the reactor chamber. Scaled experiments with relevant perveance (the ratio of space charge repulsion to ion kinetic energy) have shown that the emittance growth from the above effects can be controlled. The next goals require experiments at higher current and energy, and at driver repetition rate.

Ultimately, an integrated experiment (HIDIX) precedes a DEMO, and would, if successful, validate the HIF approach. It must demonstrate all of the key driver beam manipulations: creation of a high-current, low emittance beam, injection, matching and merging of multiple beams into the accelerator at $10 \mathrm{~Hz}$, acceleration and focusing through magnetic quadrupoles, longitudinal bunch control, and at the end of the accelerator, separation of beams at the end of the accelerator, the bunching of the beam from $\sim 100 \mathrm{~ns}$ to $\sim 10 \mathrm{~ns}$ at the target. The resulting beams will be capable of fusion-relevant target heating and compression. Several years of R\&D are required to reduce risk and to develop cost-effective components for the experiment (eg: injectors and acceleration modules).

\section{Related R\&D Activities}

- Superconducting focusing magnets, pulsed power, alignment and diagnostics for accelerators

- The research programs in heavy ion fusion in Europe, Russia, and Japan

Recent Successes

Experiments and simulations showed that stable beam transport over long distances in the 
accelerator can be achieved with low emittance growth in the presence of high space charge. Scaled experiments addressing most beam manipulations in a driver have been completed. Advanced simulations suggest that it will be possible to achieve adequate focusing at the required peak power.

- The High Current Experiment showed that beams of driver current and brightness can be created, injected and transported over short distances.

- Scaled experiments and advanced simulations addressed beam manipulations required in a driver. Most of these experiments have been scaled, using beams of 10-50 mA, in order to test critical beam physics with the relevant perveance: Test of emittance growth through many quadrupoles (SBTE), merging beams transversely while retaining good beam quality (Combiner, Multibeamlet Merging Experiment), focusing to millimeter focal spots (FFSE, $N T X, N D C X)$, experiments on beam bending $(U M E R)$. Current amplification by longitudinal compression of a few to $\approx 50$ times has been demonstrated $(M B E-4, N D C X)$.

\section{Anticipated Contributions}

\section{Metrics}

Carry out the R\&D toward a HIDIX accelerator driver, to be followed by a ignition facility and DEMO power plant. Both HIDIX and the DEMO driver must be efficient, reliable, and durable.

\section{Near Term (first $\sim 5$ years)}

- Develop a physics design for HIDIX. Continue to develop advanced source-to-target simulations.

- Commence experiments with a single beam, at driver repetition rate $(5-10 \mathrm{~Hz})$ in an accelerator long enough to evaluate the risk of emittance growth for a driver scale beam in the HIDIX. The beam energy and current will be characteristic of the front end of the HIDIX.

- Develop cost-effective injectors, quadrupole arrays, pulsers, insulators, and induction cells.

Midterm (next $\sim 10$ years)

- Complete beam physics and injector experiments at driver scale.

- Build the HIDIX and use it for beam physics, target physics, focusing, and chamber experiments.

Long Term (next $\sim 20$ years)

- Based on the HIDIX program, develop and build a fusion ignition facility.

- Upgrade the ignition facility to a demonstration power plant using the existing driver.

\section{Principal steps to a DEMO plant}

The HIDIX will be followed by rep-rated chamber experiments with high fusion yield, including experiments and demonstration of liquid wall protection. Driver reliability and economics will be a focus of the research at this stage. The DEMO will use the same driver.

\section{Proponents' Claims}

- The cost of multi-beam accelerators scale favorably with total beam energy.

- The high reliability, durability, repetition rates, and efficiency of particle accelerators carry over to the IFE application with attractive economics.

- Experiments and advanced simulations suggest that multiple beams can be created and focused with the required peak power.

\section{Critics' Claims}

- Present-day accelerator experiments for heavy ion fusion are at a small scale.

- The heavy ion driver will be expensive, and the development steps will be costly.

- Beam quality requirements will impose additional costs or require too many beams. 

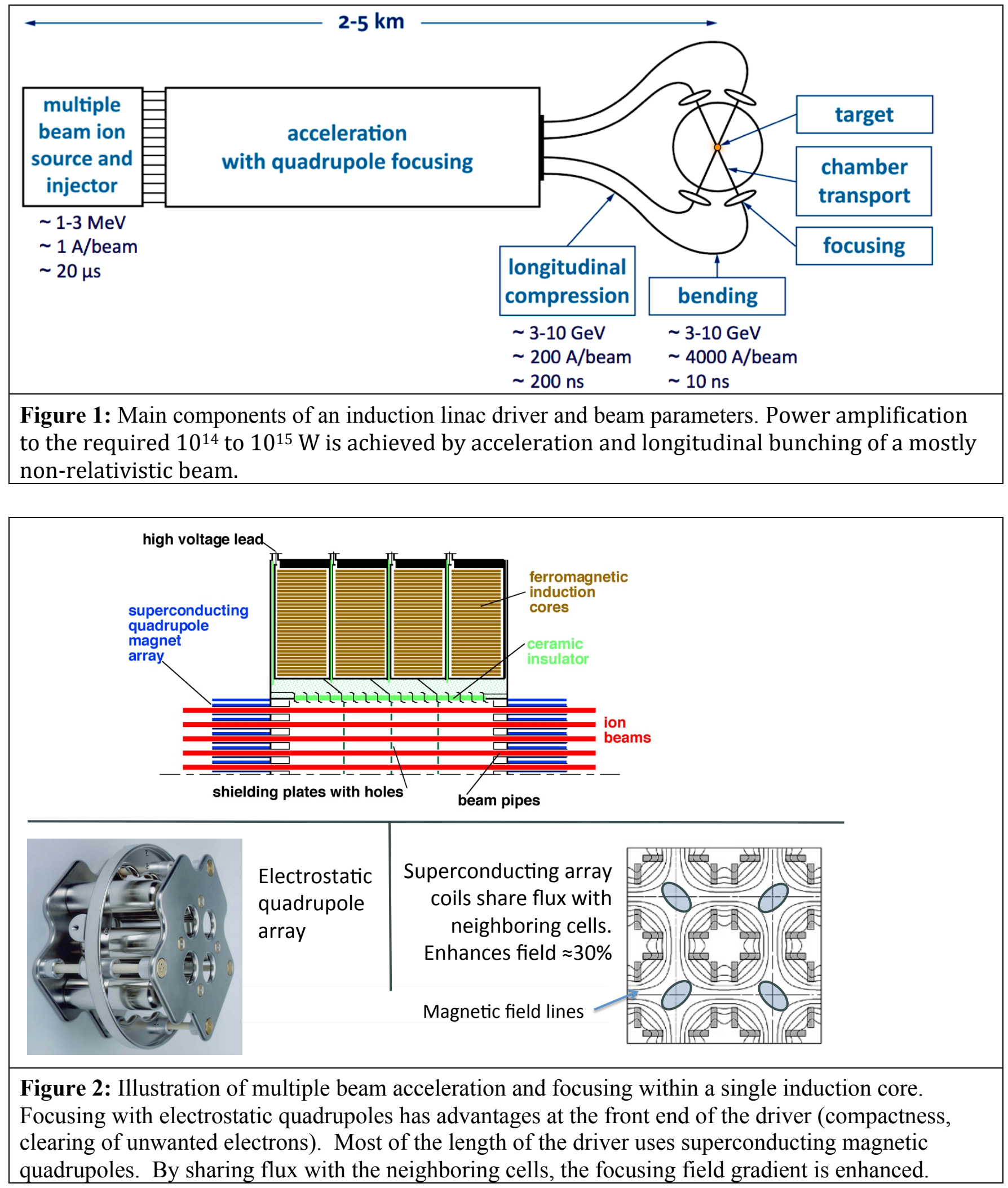
Table 1(a): Accelerators produce high-power, high energy beams. CERN's ISR had a single-beam power (protons) $1 \mathrm{TW}$ at $30 \mathrm{GeV}$. In Dec. 2010, SNS ran for 24 hours without interruption with $1 \mathrm{MW}$ beam power at $60 \mathrm{~Hz}$. Hardware availabilities of $70 \%-90 \%$ have been reached at large accelerators for high energy and nuclear physics frontier research.

\begin{tabular}{|c|c|c|c|c|}
\hline & SNS & PSI & Tevatron & LHC \\
\hline Ion species & Protons & Protons & Protons & $\mathrm{Pb}^{82+}$ \\
\hline Ion energy & $1 \mathrm{GeV}$ & $590 \mathrm{MeV}$ & $0.98 \mathrm{TeV}$ & $\begin{array}{l}2.8 \mathrm{TeV} \\
\text { /nucleon }\end{array}$ \\
\hline $\begin{array}{c}\text { Beam } \\
\text { power }\end{array}$ & $1 \mathrm{MW}$ & $1.4 \mathrm{MW}$ & & \\
\hline \multicolumn{3}{|c|}{ Stored beam energy } & $1.6 \mathrm{MJ}$ & $3.8 \mathrm{MJ}$ \\
\hline
\end{tabular}

Table 1(b): Induction linear accelerators have transported high current beam pulses, often with high repetition rate.

\begin{tabular}{|c|c|c|c|}
\hline & DARHT-II & SILUND-2 & ATA \\
\hline electron energy & $17 \mathrm{MeV}$ & $0.8 \mathrm{MeV}$ & $50 \mathrm{MeV}$ \\
\hline $\begin{array}{c}\text { Peak beam } \\
\text { current }\end{array}$ & $2 \mathrm{kA}$ & $1 \mathrm{kA}$ & $10 \mathrm{kA}$ \\
\hline Repetition rate & $<1 \mathrm{~Hz}$ & $50 \mathrm{~Hz}$ & $5 \mathrm{~Hz}$ \\
\hline
\end{tabular}

\section{Authors and Contributors}

\section{Principal Author:}

Peter A. Seidl, Lawrence Berkeley National Laboratory

\section{Co-Authors:}

John J. Barnard, Lawrence Livermore National Laboratory Richard J. Briggs, SAIC Andris Faltens, Lawrence Berkeley National Laboratory Alex Friedman, Lawrence Livermore National laboratory Igor Kaganovich, Princeton Plasma Physics Laboratory Joe Kwan, Lawrence Berkeley National Laboratory Edward P. Lee, Lawrence Berkeley National Laboratory B. Grant Logan, Lawrence Berkeley National Laboratory 


\section{Description}

- "Final optics" are here defined as the elements which focus the ion beam transversely into a small (mm scale) spot and compress it longitudinally into a short $(\sim 10 \mathrm{~ns})$ pulse at the target.

- Each ion beam in a heavy ion fusion driver will pass through a set of magnetic elements (quadrupoles and dipoles---see fig.1) whose fields focus and steer the beam onto the target. The magnet coils are not on a line-of-sight to the target, and thus avoid the direct gamma rays, ions, neutrons and target debris from the target explosion. Additional shielding prevents scattered neutrons and radiation from heating the superconducting wire or damaging the magnets, such that magnet lifetimes will be equal to or greater than the lifetime of the power plant.

- The beam is longitudinally compressed and the current pulse shape is tailored, by imposing a ramp in velocity, i.e., the tail of the beam is accelerated relative to the head. The accelerating cells that impose the ramp are far upstream, and so are easily shielded from the fusion explosion.

- In general, beam space charge acts to defocus the beam (both radially, limiting the formation of small focal spots, and longitudinally, limiting the formation of a short pulse). The baseline design employs a neutralized final focus, in which plasma electrons neutralize the beam space charge in the target chamber after the final magnetic element (a distance of order $6 \mathrm{~m}$ ). Some HIF driver designs additionally call for plasma to neutralize the beam during the final drift compression (a distance of order tens to hundreds of meters), to enhance the longitudinal compression of the beam. In such designs, plasma must be generated along the neutralized portion of the beam path. Beam interactions with vapor and plasma are discussed in "Beam-Plasma Interactions."

- In addition to the beams' own space charge, there are a number of processes that limit the spot radius. The ions' transverse thermal motion (transverse temperature) limits the minimum spot radius. Similarly, the longitudinal temperature (velocity spread) limits the minimum spot size as different ion energies have different focal lengths (chromatic aberrations). The longitudinal temperature also limits the minimum pulse duration, as different particles arrive at the target at different times. The requirement of spot size, pulse duration, pulse energy, and acceptance angle set by the target and by non-linear finite angle effects (geometric aberrations) place a constraint on the six dimensional phase space density of the beam, which by Liouville's theorem does not decrease from the injector to the target. This target acceptance limit must be consistent with the final phase space density, constrained by all sources that dilute the macroscopic phase space density from its initial value, including alignment and accelerating errors throughout the accelerator, and propagation through bends.

- For unneutralized drift compression, the beam arrives at the final focus just as the space charge force has removed the head-to-tail velocity variation, so that the final focus is nearly achromatic.

- In the chamber, multiple beams can interact through electric and magnetic fields, and final beam aiming must account for these forces.

- Final steering of beams to target is achieved by small magnetic dipole deflections on each shot.

\section{Status}

- Scaled experiments (in key dimensionless parameters, such as perveance) addressing both neutralized and unneutralized final focus have been carried out on the Final Focus Scaled Experiment (FFSE, 1999), the Neutralized Transport Experiment (NTX, 2004) and the Neutralized Drift Compression Experiment (NDCX-I, 2010). Scaled drift compression experiments have been carried out on the four-beam Multiple-Beam-Experiment (MBE-4, 1989) and NDCX-I. The NDCX-I experiment is currently exploring limits to both longitudinal and transverse compression.

- Several types of plasma generators have been employed in NTX and NDCX-I including Ferroelectric Plasma sources (FEPS) and cathodic arc plasma sources (CAPS). Plasma densities up to $\sim 10^{13} \mathrm{~cm}^{-3}$ have been achieved using the CAPS in the chamber and up to $\sim 10^{11} \mathrm{~cm}^{-3}$ have been 
achieved using the FEPS along the drift compression line, densities sufficient for beam neutralization in NDCX-I.

- The WARP and LSP 3D Particle-in-cell codes have both been employed to simulate beam propagation in a plasma, and have been validated in many experiments including the High Current Experiment (HCX), MBE-4, FFSE, NTX, NDCX-I, and the U. Md. Electron Ring (UMER).

\section{R\&D Goals and Challenges}

\section{Current Research and Development (R\&D)}

- The HIF program is currently building the Neutralized Drift Compression Experiment II (NDCX-II) that will investigate both neutralized and unneutralized drift compression and final focus. NDCX-II will ultimately achieve dimensional parameters of $\sim 1-3 \mathrm{MeV}, 30 \mathrm{~A}, 1 \mathrm{~ns}, 0.1 \mathrm{~J}$. Dimensionless parameters will meet or exceed driver parameters, e.g. final perveance (ratio of space charge potential energy to ion beam kinetic energy) $\sim 10^{-2}$ ( $c f$ driver at $10^{-4}$ to $10^{-3}$ ). Maximum plasma density in the chamber required $\sim 10^{13}$ to $10^{15} \mathrm{~cm}^{-3}$, similar to some driver designs. Modeling of multiple-beam effects will be addressed through scaled experiments, and sophisticated particle-incell codes that can simulate the ion beams, the background gas and plasma.

- Develop driver scale magnets, shielding systems and, if required, higher density plasma sources.

\section{Related R\&D Activities}

- Superconducting magnet research on final focusing systems for high-energy and nuclear physics accelerators carries over directly to Heavy Ion Fusion research.

\section{Recent Successes}

- FFSE, NTX, and NDCX-I have investigated the scaling laws of both neutralized and unneutralized final focus. MBE-4 and NDCX-I have explored un-neutralized and neutralized drift compression.

- Advanced simulations of radiation shielding of the superconducting magnets show long life.

\section{Anticipated Contributions}

\section{Metrics}

- A design for an efficient, reliable, and durable focusing system.

Near Term (first $\sim 5$ years)

- Complete the present and future scaled focusing and drift compression experiments on NDCX-I and NDCX-II (beam pulse energy 0.1 J) and its upgrade IB-HEDPX ( 1 J).

- Develop multiple beam final focus magnet arrays, plasma sources, and bend designs.

- Design the Heavy Ion Driven Implosion Experiment (HIDIX) that will employ driver-like drift compression and final focus, including multiple beam issues.

- Advanced two stage focusing systems (using plasma lenses or beam self-pinch) will be designed and studied using plasma lens and/or beam self-magnetic fields to achieve higher power densities on target, for potential use in near term experiments and drivers.

\section{Midterm (next $\sim 10$ years)}

- Build the HIDIX and use it for focusing, drift compression and chamber experiments ( $\sim 10 \mathrm{~kJ})$.

- After completing the HIDIX program, develop and build a full-scale ignition facility.

Long Term (next $\sim 20$ years)

- Upgrade the ignition facility to a demonstration power plant using the existing driver $(\sim \operatorname{several~MJ)}$

Principal steps to a DEMO plant
$\mathrm{NDCX}-\mathrm{II} \rightarrow \mathrm{HIDIX} \rightarrow \mathrm{HIF}$ ignition facility $\rightarrow$ upgrade to DEMO.

\section{Proponents' Claims}

Proponents claim that the ability to shield the final focusing optics from photons, neutrons, ions and debris generated by the target implosion, is a distinct and clear advantage for heavy ion fusion. The research program outlined above addresses critics claims stated below.

\section{Critics' Claims}

Critics claim that the small scale of present-day final focus experiments is insufficient to validate claims that small focal spots can be achieved at driver scale, using multiple beams. 

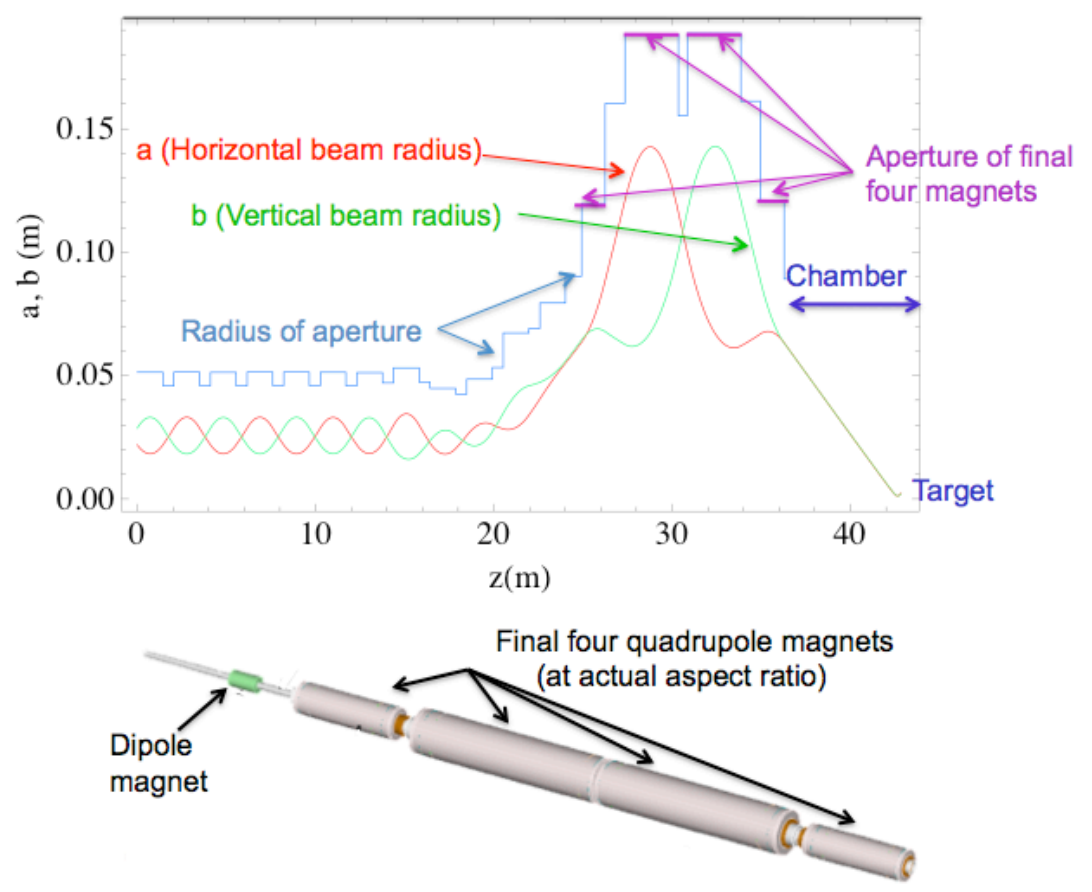

Figure 1. Upper: Horizontal and vertical beam radius as the beam passes through final few quadrupoles in a final focus system. Lower: Sketch of the final four magnets with actual aspect ratio shown.

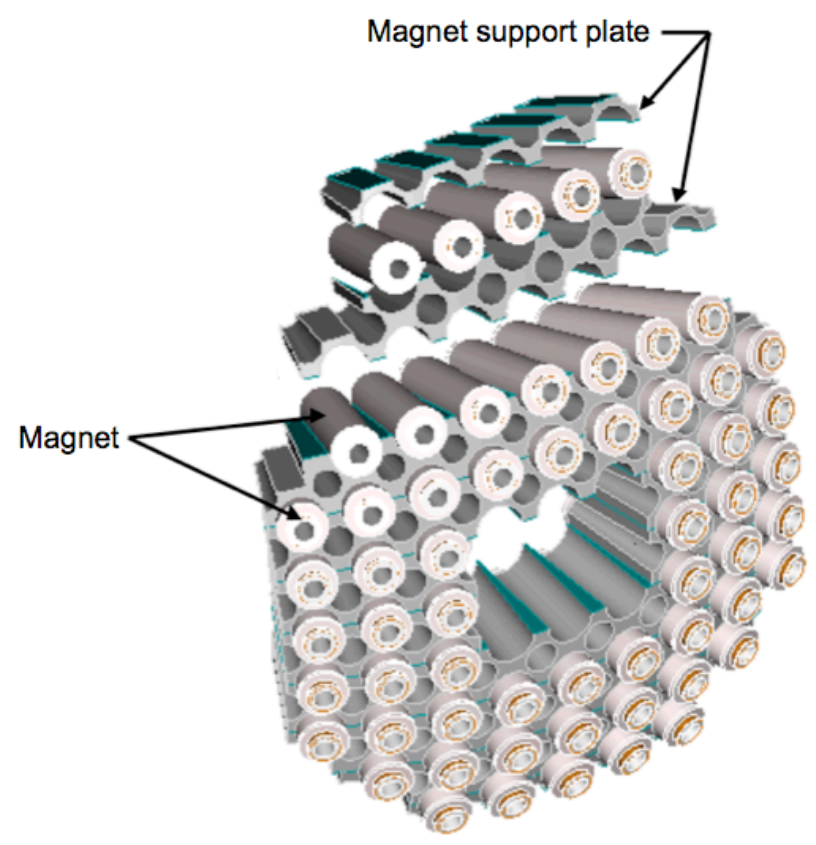

Figure 2. Magnet system support and assembly concept for final focus array of the final magnet of the Robust Point Design study. Central hole facilitates target injection. 


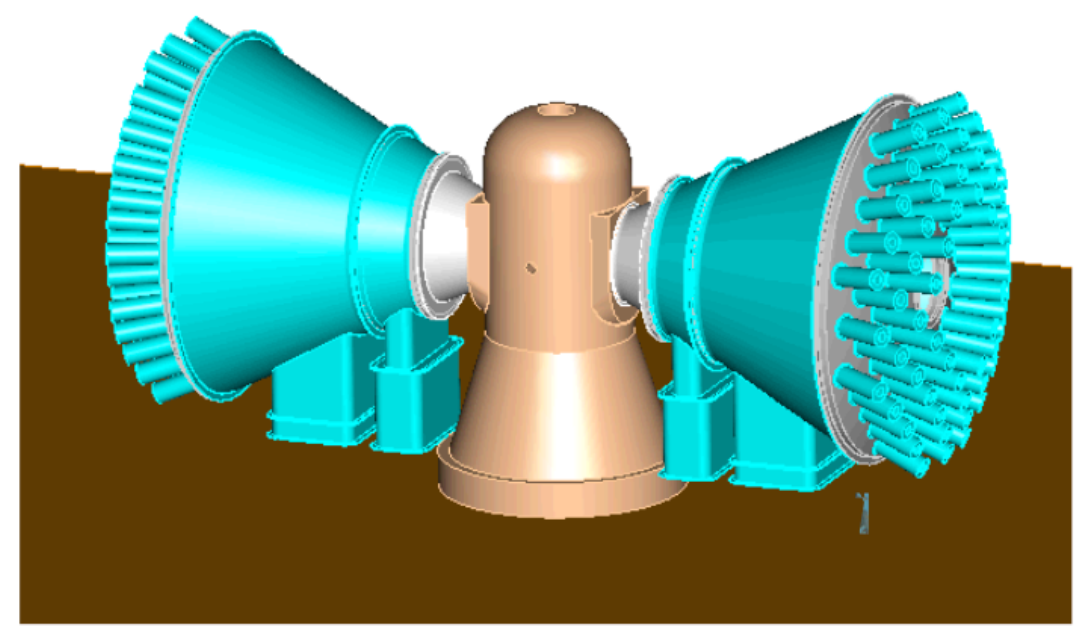

Figure 3. Layout of the final beamlines and the chamber in the 2002 Robust Point Design study.

\section{Authors and Contributors}

\section{Principal Author:}

John J. Barnard, Lawrence Livermore National Laboratory

\section{Co-Authors:}

Andris Faltens, Lawrence Berkeley National Laboratory Alex Friedman, Lawrence Livermore National laboratory Igor Kaganovich, Princeton Plasma Physics Laboratory Joe Kwan, Lawrence Berkeley National Laboratory B. Grant Logan, Lawrence Berkeley National Laboratory Peter A. Seidl, Lawrence Berkeley National Laboratory 


\section{BEAM PLASMA INTERACTIONS}

\section{Description}

In Heavy Ion Fusion, intense beams of ions from an accelerator must deliver their energy into compact (mm-scale) volumes within fusion targets. A variety of beam-plasma interactions arise:

- In the target (Fig. 1), the ion beams interact with matter (radiation converters or ablator material) that stops them and absorbs their kinetic energy, using it to (indirectly or directly) drive the implosion and ignition of the fuel capsule. This matter rapidly becomes dense plasma.

- In the fusion chamber (Figs 2 and 3), plasma is created when ion beams and target radiation ionize the background vapor (which is in the mTorr pressure range). Plasma can also be provided by external sources (or a pre-pulse hitting a target extension) to aid neutralizedballistic focusing, wherein plasma electrons adjust their distribution to cancel out the macroscopic space charge field of the ions.

- In some concepts (though not the baseline), there is a need for a neutralizing plasma in, or near the end of, the drift compression section (between the accelerator and final optic), to enable use of "neutralized drift compression" so as to obtain shorter beam pulses.

- In the accelerator, the impact of outlying ("halo") beam ions on the wall causes the emission of "secondary" electrons. The resulting "electron cloud" may interact with the beam.

\section{Status}

- Ion stopping in the target is mainly due to interactions with bound electrons in atoms and ions. Stopping and scattering in solid and gas targets is well-studied; data are available for a variety of ions and target materials. In plasma, the ion range is shortened by the contributions of free electrons. For classical plasma, mature theory exists and is being benchmarked by experiments at GSI shooting ions into laser-heated matter at high temperature and low density. This effect must be accounted for in target design; indeed, it allows reduction of the mass of the heated matter. There is no analogue to the stimulated scattering seen in laser-target interactions.

- In the fusion chamber, the plasma density must be large relative to the beam density for effective charge neutralization and a compact focal spot. This process is robust, and has been employed on recent experiments at LBNL. The cross-sections for beam stripping and charge exchange on background atoms or ions have been extensively studied experimentally and theoretically. Beam-plasma interactions have been extensively studied for a variety of applications. Collective processes may include focusing associated with polarization of the background plasma, interactions among the beams in plasma, and unstable modes such as hose, sausage, filamentation, and two-stream which must be controlled by design. The latter are detuned or inhibited by the variation of beam parameters along the pulse, the finite transit time in the chamber, the large inertia of heavy ions, and the inhomogeneity of the beam and plasma profiles. Enhanced focusing may be achievable via self-pinch effects or via a plasma lens (as at the GSI and ITEP-TWAC ion beam facilities in Germany and Russia). Propagation in the chamber is understood using experiments, theory, and advanced particle-in-cell simulations.

- Neutralized drift compression (NDC) allows a much shorter final pulse than can be obtained using the baseline non-neutral drift compression. In non-neutral drift compression, spacecharge repulsion ultimately limits bunch compression but yields a nearly-monoenergetic beam at the final optic, minimizing chromatic aberrations and making it easier to achieve a small beam spot on target. NDC is the basis for the NDCX-I and the NDCX-II facility now under construction. It is highly effective provided that the plasma density exceeds the beam density.

- In the accelerator, the impact of halo beam ions on the pipe wall induces the emission of a lowdensity electron cloud, which could influence beam dynamics through their space charge, and neutral atoms which raise the background gas pressure. Halo ions can be scraped away, and 
stray electrons can be swept out by strong electric and magnetic fields in accelerating and focusing (confining) elements.

\section{R\&D Goals and Challenges \\ Current Research and Development (R\&D)}

- Validate theory of beam transport and focusing for high current beams in plasma.

- Develop plasma sources compatible with the chamber and drift line. These should have high plasma density, low gas pressure, and the ability to operate at the repetition rate of a reactor.

- Further assess beam emittance growth during propagation in plasma for driver scale beams.

- Further develop advanced 3D numerical simulation capability for beam-plasma interactions.

- Evaluate beam focusing and steering in plasma by application of external magnetic field.

Related R\&D Activities

- Energetic particle interaction with plasma in magnetic fusion, space plasmas, astrophysics.

- Generation of intense particle beams by lasers for various applications.

- Wake-field and other collective acceleration schemes using beam plasma-interactions.

Recent Successes

- Completion of neutralized drift and focusing experiments demonstrating large compressions.

- Construction and successful operation of several-meters-long ferroelectric plasma source.

- Demonstration of collective acceleration in experiments at SLAC, LBNL, and elsewhere.

- Solid progress toward validated, advanced 3D PIC codes for beam-plasma interaction.

\section{Anticipated Contributions}

\section{Metrics}

- An efficient, reliable, durable, plasma neutralization and focusing system that meets IFE system requirements, having adequate $(5-10 \mathrm{~Hz})$ repetition rate and cost.

Near Term (first $\sim 5$ years)

- Operate NDCX-II to validate NDC and focusing for larger beam space charge than NDCX-I.

- Further assess impact of beam-plasma instabilities on emittance.

- Evaluate neutralized drift compression scheme for negative ion beams.

- Upgrade the HCX facility at LBNL to carry out quantitative studies of beam interactions with residual gas and stray electron cloud, at $5-10 \mathrm{~Hz}$ and over relevant distance.

- Further develop detailed simulation capabilities and benchmark vs. the above experiments.

Midterm (next $\sim 10$ years)

- Evaluate neutralized transport for HIDIX facility and a full-scale driver.

Long Term (next $\sim 20$ years)

- Validate neutralized transport for a full-scale driver, at rep rate.

\section{Principal steps to a DEMO plant}

- Validate feasibility of neutralized transport for all steps leading to full-scale driver.

\section{Proponents' Claims}

- Neutralized ion beam transport in the chamber is compatible with a thick liquid wall.

- Beam space charge is well-neutralized by a background plasma of suitable density.

- Beam plasma instabilities are mitigated due to finite transit time through the chamber, large inertia of heavy ions, and inhomogeneity of the beam and plasma density profiles;

- Electron cloud effects are mitigated by applied electric and magnetic fields, and beam control.

\section{Critics' Claims}

- Plasmas introduce beam and background plasma dynamics, including the following: streaming and filamentation instabilities, imperfect neutralization, beam stripping, emittance growth. 


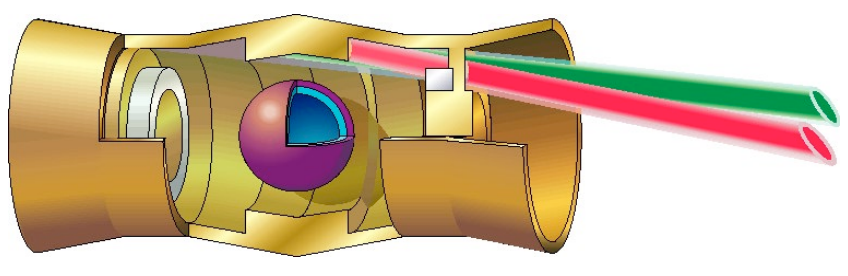

(a)

(b)

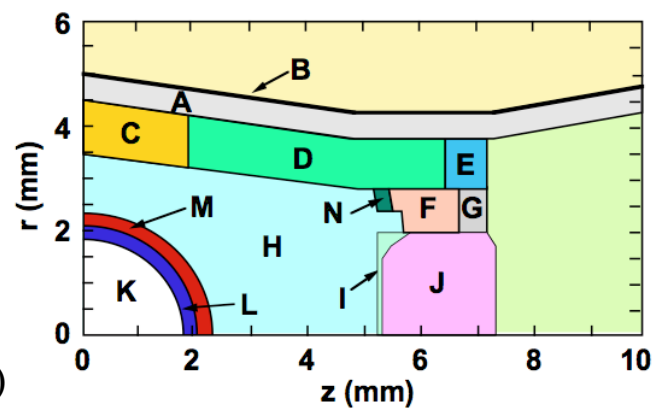

Fig. 1. (a) Indirect-drive HIF target [from D. Callahan-Miller and M. Tabak, Phys. Plasmas 7 (2000)]; (b) schematic showing beam absorbing elements at, e.g., positions $\mathrm{C}$ (Fe at $0.016 \mathrm{~g} / \mathrm{cc}$ ) and $\mathrm{D}\left([\mathrm{CH}]_{0.97} \mathrm{Au}_{0.03}\right.$ at $\left.0.011 \mathrm{~g} / \mathrm{cc}\right)$ [from D. Goodin's target-fabrication presentation to the NAS-IFE panel on Jan 30, 2011, available at http://fire.pppl.gov/icf_nas_review_2010.html ].

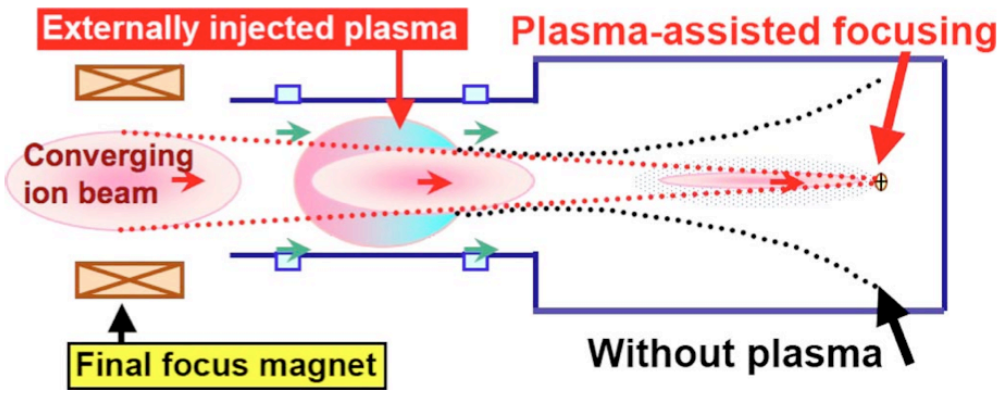

Fig. 2. Plasma-assisted transverse focusing, wherein neutralization allows to beam to focus to a compact (emittance-limited) spot [from A. Sefkow, et al., Phys. Plasmas 16, 056701 (2009)].

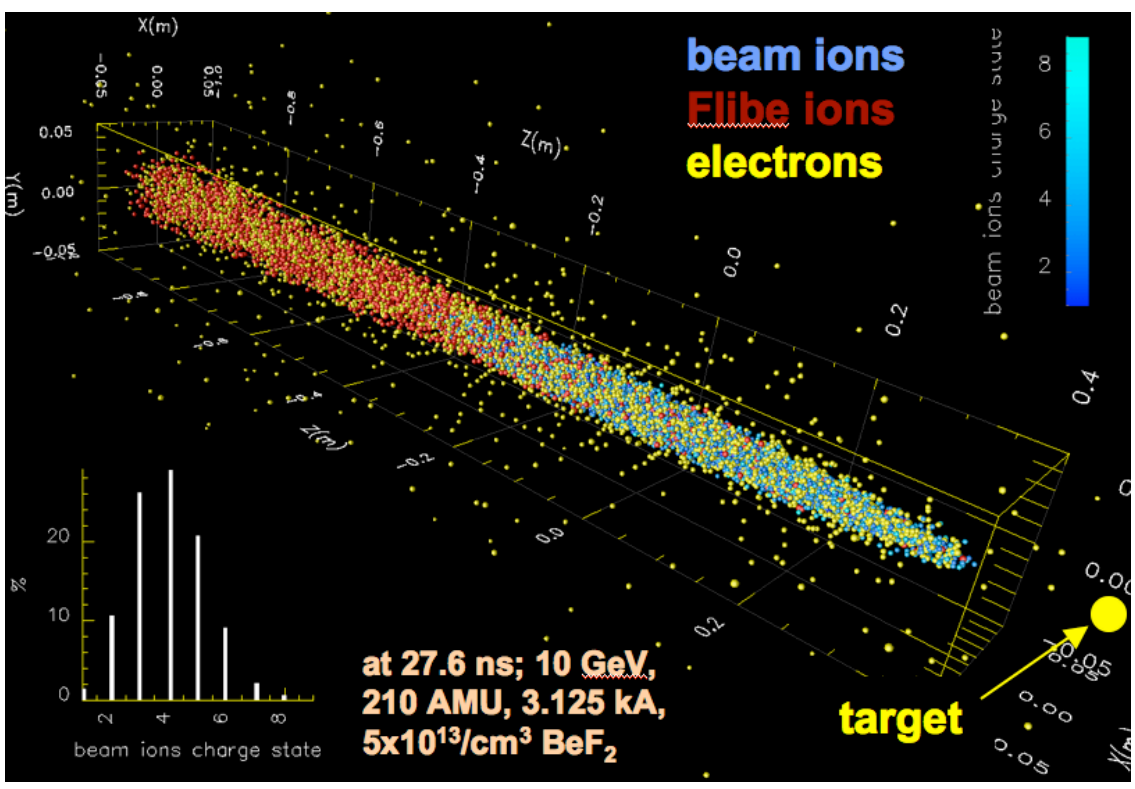

Fig. 3. Snapshot from a particle-in-cell simulation of neutralized ion beam transport in chamber, showing beam and background ions, electrons, and distribution of beam ion charge states. 


\section{Authors and Contributors}

\section{Principal Author:}

Igor Kaganovich, Princeton Plasma Physics Laboratory

\section{Co-Authors:}

John J. Barnard, Lawrence Livermore National Laboratory Alex Friedman, Lawrence Livermore National laboratory B. Grant Logan, Lawrence Berkeley National Laboratory Peter A. Seidl, Lawrence Berkeley National Laboratory

\section{Contributors:}

Andris Faltens, Lawrence Berkeley National Laboratory Edward P. Lee, Lawrence Berkeley National Laboratory Richard M. More, Lawrence Berkeley National Laboratory

Erik Gilson, Princeton Plasma Physics Laboratory Larry Grisham, Princeton Plasma Physics Laboratory Ed Startsev, Princeton Plasma Physics Laboratory

Dale R. Welch, Voss Scientific

Dave Sutter, University of Maryland 